\title{
Elastic Reciprocity and Symmetry Constraints on the Stress Field Due to a Surface-Parallel Distribution of Dislocations
}

\section{Citation}

Viesca, Robert C. and James R. Rice. 2011. Elastic reciprocity and symmetry constraints on the stress field due to a surface-parallel distribution of dislocations. Journal of the Mechanics and Physics of Solids 59(4): 753-757.

\section{Published Version}

doi:10.1016/j.jmps.2011.01.011

\section{Permanent link}

http://nrs.harvard.edu/urn-3:HUL.InstRepos:5026689

\section{Terms of Use}

This article was downloaded from Harvard University's DASH repository, and is made available under the terms and conditions applicable to Open Access Policy Articles, as set forth at http:// nrs.harvard.edu/urn-3:HUL.InstRepos:dash.current.terms-of-use\#OAP

\section{Share Your Story}

The Harvard community has made this article openly available.

Please share how this access benefits you. Submit a story.

\section{Accessibility}


Publication reference: Journal of the Mechanics and Physics of Solids, volume 59, issue 4, pages 753-757, April 2011, doi:10.1016/ j.jmps.2011.01.011 (original manuscript submitted 26 August 2010, accepted 19 January 2011, available online 27 January 2011).

\title{
Elastic reciprocity and symmetry constraints on the stress field due to a surface-parallel distribution of dislocations
}

\author{
Robert C. Viesca ${ }^{a}$, James R. Rice ${ }^{\mathrm{a}, \mathrm{b}}$ \\ ${ }^{a}$ School of Engineering and Applied Sciences, Harvard University, Cambridge, MA 02138 \\ ${ }^{b}$ Department of Earth and Planetary Sciences, Harvard University, Cambridge, MA \\ 02138
}

\begin{abstract}
Elastic reciprocity and geometric symmetry are used to constrain the expressions for stresses due to introduction of line dislocations near a half-space surface. Specifically, a relationship is shown to exist between the changes induced by dislocations of orthogonal Burgers vectors (normal and parallel to the free surface). These results are used to address inconsistencies of solutions in the literature.
\end{abstract}

Keywords:

dislocation, half-space, elastic, reciprocity

\section{Background}

There is currently a variance in the literature regarding the solution for an edge dislocation near a free surface which has persisted for more than 40 years. With reference to Figure 1, the point of disagreement is whether, at a point along the $x$ axis, the change of the surface-normal stress $\sigma_{y y}$ due to the dislocation of Figure 1a is opposite or equal to the change of shear stress $\sigma_{y x}$ due to the dislocation of Figure 1b, for the same magnitude of Burgers vector (displacement discontinuity) in both 1a and 1b. The original solution of the dislocation problem by Head (1953), consistently with an independent solution by Dundurs and Sendecky (1965), indicated the relation to be opposite (as drawn in Figure 1). However, among his influential body of work on reducing crack and contact problems to singular integral equations and devising effective numerical solution techniques, one special case 
addressed by Erdogan (1969) required that same dislocation solution. His result, repeated in Erdogan et al. (1973), can be seen to match the original Head solution with the exception of a difference of sign in one term. That difference indicates the relation discussed above to, instead, be equal.

This discrepancy became apparent when examining the stress intensity factors for a uniformly pressurized subsurface crack, represented as a continuous distribution of such dislocations. These factors were first numerically calculated in the case of the equal relation by Erdogan (1969) and Erdogan et al. (1973), and in that of the opposite relation by Ashbaugh (1975), who first suggested the solution in Erdogan et al. (1973) required correction. Subsequently, Chen et al. (1980) also suggested the same correction, and, in addition to Higashida and Kamada (1982), also calculated the intensity factors in agreement with those of Ashbaugh (1975). It is the results of Higashida and Kamada (1982) that feature ambiguously alongside and in contrast with those of Erdogan et al. (1973) in a handbook of stress intensity solutions (Murakami, 1987, p. 167).

However, there are indications that one of the solutions may be correct. Examining the stress intensity factors, $K_{I}$ and $K_{I I}$, in the limit of a very shallow crack using a beam approximation, Dyskin et al. (2000) note agreement with Higashida and Kamada (1982) over Erdogan et al. (1973). Additionally, several other authors have presented consistent solutions (Thouless et al., 1987; Yang and Li, 1997; the latter claim a misprint in the solution of Head, 1953, although we find no such misprint when comparing their solutions). Additionally, work by Erdogan (1971) for a crack parallel to a bimaterial interface between joined half-spaces indicates an opposite relation. Because that configuration reduces, in the limit of zero stiffness for the uncracked halfspace, to the problem considered in 1969 and 1973, there is an inconsistency among the set of papers. Further, Tada et al. (2000, p. 231) give a beam theory asymptote for the mode I stress intensity factor that is different from Dyskin et al. (2000) and seemingly in agreement with the results of Erdogan et al. (1973). However, if we assume that beam theory adequately describes energy transfer to the crack tip, hence implies $K_{I}^{2}+K_{I I}^{2}$, that mode-I asymptote requires that $K_{I I}=\sqrt{3} K_{I}$, which deviates strongly from the trend in mode II of Erdogan et al. (1973). Furthermore, Paynter et al. (2006), aware of the sign mismatch leading to the dissimilar results, maintain that the solution of Erdogan et al. (1973) is correct. The matter appears to some extent unsettled and we aim here to conclusively address the discrepancy based not on re-deriving specific proposed solutions but, rather, appealing to general 

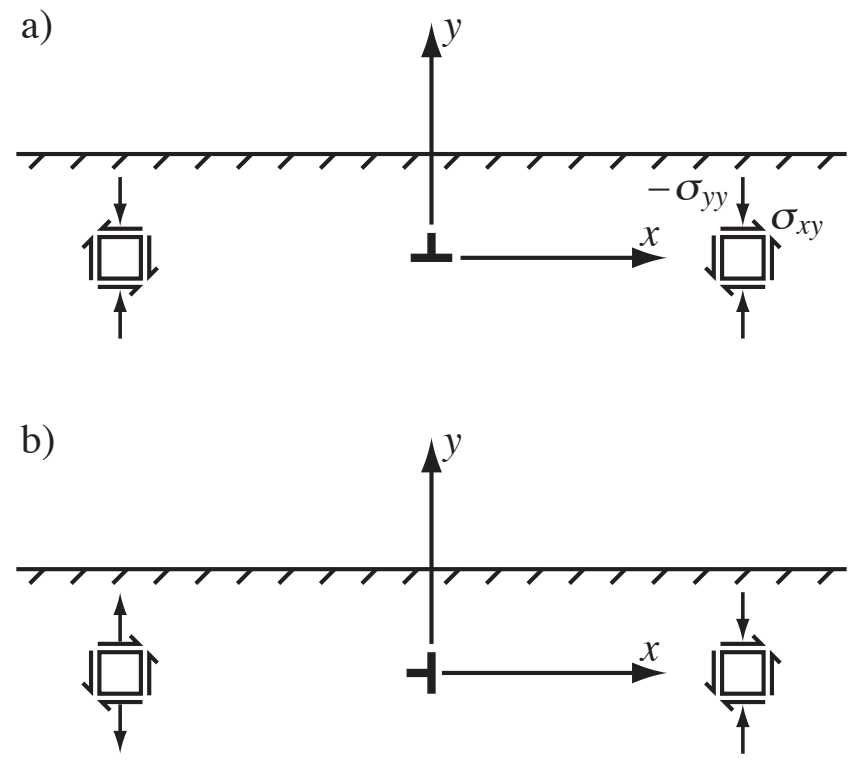

Figure 1: Elements along the $x$-axis and at symmetric points about the $y$-axis indicate stress $\left(\sigma_{x x}\right.$ omitted) due to an edge dislocation with Burgers vector a) parallel and b) perpendicular to a free surface a distance $h$ away. Standard positive conventions for normal (in tension) and shear stresses are designated in a). 
considerations of elastic reciprocity and symmetry.

\section{Elastic reciprocity}

The Betti reciprocal theorem requires that for two elastic stress-displacement fields $\sigma_{\imath \jmath}^{(1)}, u_{k}^{(1)}$ and $\sigma_{\imath \jmath}^{(2)}, u_{k}^{(2)}$ in the half space, corresponding to different distributions of displacement discontinuity along a cut (e.g., to represent a crack) coincident with a portion of the $x$ axis (which is parallel to the free surface), that

$$
\int_{S}\left(n_{\imath} \sigma_{\imath \jmath}^{(1)}\right) u_{\jmath}^{(2)} d S=\int_{S}\left(n_{\imath} \sigma_{\imath \jmath}^{(2)}\right) u_{\jmath}^{(1)} d S
$$

Here the origin of the $y$-axis is a distance $h$ below the free surface, and the closed contour $S$ runs along $y=0^{ \pm}$along the length of the cut. We consider cases of balanced tractions along $y=0^{ \pm}$so that, for $\imath=x, y$, $\sigma_{y \imath}\left(x, 0^{+}\right)=\sigma_{y \imath}\left(x, 0^{-}\right) \equiv \sigma_{y \imath}(x)$ along that contour. Then, defining displacement discontinuities $\delta_{\imath}(x) \equiv u_{\imath}\left(x, 0^{+}\right)-u_{\imath}\left(x, 0^{-}\right)$along the cut, reciprocity requires

$$
\begin{array}{r}
\int_{-\infty}^{\infty}\left[\sigma_{y x}^{(1)}(x) \delta_{x}^{(2)}(x)+\sigma_{y y}^{(1)}(x) \delta_{y}^{(2)}(x)\right] d x= \\
\int_{-\infty}^{\infty}\left[\sigma_{y x}^{(2)}(x) \delta_{x}^{(1)}(x)+\sigma_{y y}^{(2)}(x) \delta_{y}^{(1)}(x)\right] d x
\end{array}
$$

(with the integrands vanishing here and below for positions $x$ outside the cut).

The stresses $\sigma_{y \imath}(x)$ due to continuous distributions of dislocations of the types in Figure 1a and b, with local densities $-d \delta_{k}(x) / d x$ over the domain $-\infty<x<\infty$, have the form

$$
\sigma_{y \imath}(x)=\frac{\mu}{2 \pi(1-\nu)} \int_{-\infty}^{\infty}\left[\frac{1}{t-x} \frac{d \delta_{\imath}(t)}{d t}+K_{\imath \jmath}(t-x) \frac{d \delta_{\jmath}(t)}{d t}\right] d t
$$

While labeling the kernels as $K_{\imath \jmath}(t-x)$ versus a notation $L_{\imath \jmath}(x-t)$ is an arbitrary and inconsequential choice, we have chosen to follow the implicit preference of Erdogan (1969). 
Choosing $\delta_{x}^{(2)}(x)=0, \delta_{y}^{(1)}(x)=0$ and substituting the relations for stress

$$
\begin{array}{r}
\int_{-\infty}^{\infty}\left[\int_{-\infty}^{\infty} K_{y x}(t-x) \frac{d \delta_{x}^{(1)}(t)}{d t} d t\right] \delta_{y}^{(2)}(x) d x= \\
\int_{-\infty}^{\infty}\left[\int_{-\infty}^{\infty} K_{x y}(t-x) \frac{d \delta_{y}^{(2)}(t)}{d t} d t\right] \delta_{x}^{(1)}(x) d x
\end{array}
$$

Integrating the innermost integrals by parts, noting that the kernels must vanish as $|t-x| \rightarrow \infty$, and assuming that $\delta_{x}^{(1)}(t), \delta_{y}^{(2)}(t)$ are bounded as $t \rightarrow \pm \infty$

$$
\begin{array}{r}
\int_{-\infty}^{\infty}\left[\int_{-\infty}^{\infty} K_{y x}^{\prime}(t-x) \delta_{x}^{(1)}(t) d t\right] \delta_{y}^{(2)}(x) d x= \\
\int_{-\infty}^{\infty}\left[\int_{-\infty}^{\infty} K_{x y}^{\prime}(t-x) \delta_{y}^{(2)}(t) d t\right] \delta_{x}^{(1)}(x) d x
\end{array}
$$

where $K_{\imath \jmath}^{\prime}(\theta)=d K_{\imath \jmath}(\theta) / d \theta$. Switching the symbols for the integration variables $x$ and $t$ on the left hand side and rearranging

$$
\int_{-\infty}^{\infty} \int_{-\infty}^{\infty} \delta_{x}^{(1)}(x) \delta_{y}^{(2)}(t)\left[K_{x y}^{\prime}(t-x)-K_{y x}^{\prime}(x-t)\right] d t d x=0
$$

That equation must be satisfied for an arbitrary choice of the functions in the product $\delta_{x}^{(1)}(x) \delta_{y}^{(2)}(t)$, requiring that

$$
K_{x y}^{\prime}(t-x)=K_{y x}^{\prime}(x-t)
$$

Then noting that $K_{x y}^{\prime}(\theta)-K_{y x}^{\prime}(-\theta)=0$ is equivalent to $d\left[K_{x y}(\theta)+K_{y x}(-\theta)\right] / d \theta=$ 0 , we conclude that

$$
K_{x y}(t-x)=-K_{y x}(x-t)
$$

\section{Symmetry}

To further sharpen the constraint of Equation 8, consider the two cases

$$
\delta_{x}^{(1)}(x)=-\frac{b_{x}}{2} \frac{x}{|x|}, \quad \delta_{y}^{(2)}(x)=-\frac{b_{y}}{2} \frac{x}{|x|} \quad \text { for } \quad|x|>0
$$

with $\delta_{x}^{(1)}(0)=\delta_{y}^{(2)}(0)=0$, and with $\delta_{y}^{(1)}(x)=\delta_{x}^{(2)}(x)=0$ for all $x$. These describe a classical edge dislocation line running perpendicular to the $x, y$ 
plane through its origin, i.e., along the $z$ direction, with Burgers vector components $b_{x}$ in case (1) and $b_{y}$ in case (2). The stress field created in case (1) is equivalent to that produced by insertion of a sliver of material of thickness $b_{x}$ along the portion of the $y, z$ plane corresponding to $y>0$ (Figure 1a). That created in case (2) is equivalent to that produced by insertion of a sliver of thickness $b_{y}$ along the portion of the $x, z$ plane corresponding to $x<0$ (Figure 1b).

The resulting stresses on $y=0$ in the two cases are, by Equation 3,

$$
\begin{aligned}
& \sigma_{y x}^{(1)}(x)=\frac{\mu b_{x}}{2 \pi(1-\nu)}\left[\frac{1}{x}-K_{x x}(-x)\right], \quad \sigma_{y y}^{(1)}(x)=-\frac{\mu b_{x}}{2 \pi(1-\nu)} K_{y x}(-x) \\
& \sigma_{y x}^{(2)}(x)=-\frac{\mu b_{y}}{2 \pi(1-\nu)} K_{x y}(-x), \quad \sigma_{y y}^{(2)}(x)=\frac{\mu b_{y}}{2 \pi(1-\nu)}\left[\frac{1}{x}-K_{y y}(-x)\right]
\end{aligned}
$$

Case (1) has mirror symmetry about the $y, z$ plane, $u_{x}^{(1)}(x, y)=-u_{x}^{(1)}(-x, y)$, $u_{y}^{(1)}(x, y)=u_{y}^{(1)}(-x, y)$, so that $\sigma_{y x}^{(1)}(+x)=-\sigma_{y x}^{(1)}(-x)$ and $\sigma_{y y}^{(1)}(+x)=$ $\sigma_{y y}^{(1)}(-x)$, assuring that

$$
K_{x x}(-x)=-K_{x x}(+x) \quad \text { and } \quad K_{y x}(-x)=K_{y x}(+x)
$$

Case (2) has pure antisymmetry about that $y, z$ plane, requiring that $u_{x}^{(2)}(x, y)=$ $u_{x}^{(2)}(-x, y), u_{y}^{(2)}(x, y)=-u_{y}^{(2)}(-x, y)$, so that $\sigma_{y x}^{(2)}(+x)=\sigma_{y x}^{(2)}(-x)$ and $\sigma_{y y}^{(2)}(+x)=-\sigma_{y y}^{(2)}(-x)$, assuring that

$$
K_{x y}(-x)=K_{x y}(+x) \quad \text { and } \quad K_{y y}(+x)=-K_{y y}(-x)
$$

Thus both $K_{x y}(t-x)$ and $K_{y x}(t-x)$ are even functions of $t-x$ and our previous deduction that $K_{x y}(t-x)=-K_{y x}(x-t)$ can be rewritten as

$$
K_{x y}(t-x)=-K_{y x}(t-x)
$$

Reverting to the opening discussion, this result, based only on elastic reciprocity and symmetry, clearly shows that the opposite alternative must be the correct one. This result is not restricted to the coordinate choice of Figure 1, as we show next.

\section{Coordinate changes}

Given $K_{\imath \jmath}(\theta)$ for a particular choice of coordinates $x, y$, the components $K_{\tilde{\imath}}(\theta)$ may be determined for an alternative choice $\tilde{x}, \tilde{y}$. Consider the coordinates $\tilde{x}=-x, \tilde{y}=-y$ (e.g., Erdogan, 1969; Erdogan et al., 1973; Figure 2), 
a)

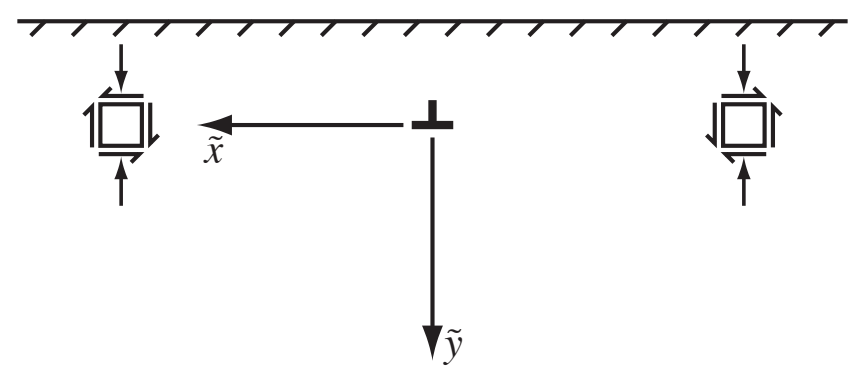

b)

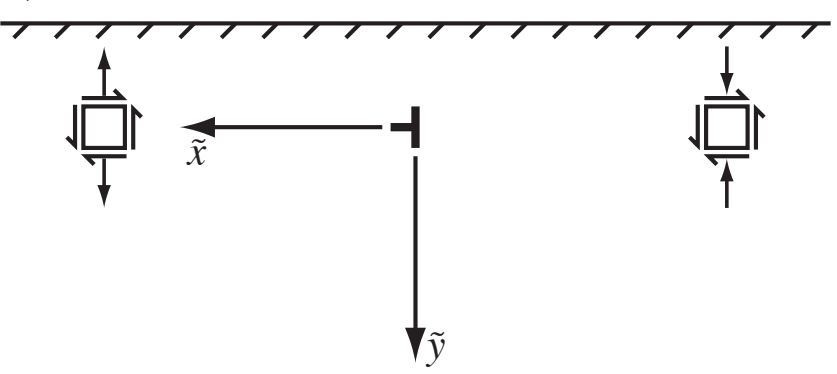

Figure 2: Elements along the $\tilde{x}$-axis and at symmetric points about the $\tilde{y}$-axis indicate stress $\left(\sigma_{\tilde{x} \tilde{x}}\right.$ omitted) due to an edge dislocation with Burgers vector a) parallel and b) perpendicular to a free surface a distance $h$ away. 
for which the displacement discontinuities are $\delta_{\tilde{\imath}}(\tilde{x})=u_{\tilde{\imath}}^{+}\left(\tilde{x}, 0^{+}\right)-u_{\tilde{\imath}}^{-}\left(\tilde{x}, 0^{-}\right)$ for $\tilde{\imath}=\tilde{x}, \tilde{y}$, and the stress components take the form

$$
\sigma_{\tilde{y} \tilde{\imath}}(\tilde{x})=\frac{\mu}{2 \pi(1-\nu)} \int_{-\infty}^{\infty}\left[\frac{1}{\tilde{t}-\tilde{x}} \frac{d \delta_{\tilde{\imath}}(\tilde{t})}{d \tilde{t}}+K_{\tilde{\imath} \tilde{\jmath}}(\tilde{t}-\tilde{x}) \frac{d \delta_{\tilde{\jmath}}(\tilde{t})}{d \tilde{t}}\right] d \tilde{t}
$$

We note that $\delta_{\tilde{x}}(-x)=\delta_{x}(x)$ and $\delta_{\tilde{y}}(-x)=\delta_{y}(x)$ and that $\sigma_{\tilde{y} \tilde{x}}(-x)=\sigma_{y x}(x)$, and $\sigma_{\tilde{y} \tilde{y}}(-x)=\sigma_{y y}(x)$. Using these relations for displacement discontinuities and stress components between the two coordinate systems, the coordinate transformations themselves, and the anticipated even or odd nature of $K_{\tilde{\imath} \tilde{\jmath}}(\theta)$, we may transform the expression for $\sigma_{\tilde{y} \tilde{\imath}}(\tilde{x})$ as an integral over $\tilde{t}$ (Equation 15) to an expression for $\sigma_{y \imath}(x)$ as an integral over $t$. Doing so and comparing

the resulting expression with Equation 3, we find the relation between the nonsingular kernels: $K_{\imath \jmath}(\theta)=K_{\tilde{\imath} \tilde{\jmath}}(\theta)$ for $\imath=\jmath$ and $K_{\imath \jmath}(\theta)=-K_{\tilde{\imath} \jmath}(\theta)$ for $\imath \neq \jmath$. (Here the notation is such that if $\imath=x$, then $\tilde{\imath}=\tilde{x}$, etc.) That both $K_{x y}(\theta)$ and $K_{y x}(\theta)$ transform into the new coordinate system with the same sign preserves the conclusion of the previous section: $K_{\tilde{x} \tilde{y}}(\theta)=-K_{\tilde{y} \tilde{x}}(\theta)$.

This indicates that the solution proposed in Erdogan (1969) and Erdogan et al. (1973), in which $K_{\tilde{x} \tilde{y}}(\theta)=K_{\tilde{y} \tilde{x}}(\theta)$, must require correction, presumably due to an unnoticed sign error in transcription from notes or in derivation. From the above analysis alone, it is not possible to determine which of the two terms requires a sign change to arrive at $K_{\tilde{x} \tilde{y}}(\theta)=-K_{\tilde{y} \tilde{x}}(\theta)$. However, using a correct solution in another coordinate set (e.g., Head, 1953) and the coordinate transformation above, the appropriate sign change may be determined (see Appendix).

\section{Acknowledgment}

Our interest in clarifying ambiguities in the literature arose in studying models for fractures running parallel to surfaces of half-spaces as they arise in landslide and glacial mechanics, those studies being supported in the former application area by a Cooperative Research Agreement with Total E\&P Recherche Developpement under award number FR00003219, and in the latter by NSF Office of Polar Programs award ANT-0739444. We are grateful to Victor C. Tsai for discussion. 


\section{Appendix A. Expressions for stress due to edge dislocations under change of coordinates, Burgers vector convention}

For the coordinates of Figure 1 and the positive Burgers vector convention represented by cases (1) and (2) (e.g., as in Thouless et al., 1987), stresses along $y=0$ are

$$
\begin{gathered}
\frac{\sigma_{y x}^{(1)}(x)+\sigma_{y x}^{(2)}(x)}{\mu /[2 \pi(1-\nu)]=b_{x}}\left[\frac{1}{x}-\frac{x}{4 h^{2}+x^{2}}+\frac{8 h^{2} x}{\left(4 h^{2}+x^{2}\right)^{2}}+\frac{4 h^{2} x^{3}-48 h^{4} x}{\left(4 h^{2}+x^{2}\right)^{3}}\right]+ \\
b_{y}\left[\frac{24 h^{3} x^{2}-32 h^{5}}{\left(4 h^{2}+x^{2}\right)^{3}}\right] \\
\frac{\sigma_{y y}^{(2)}(x)+\sigma_{y y}^{(1)}(x)}{\mu /[2 \pi(1-\nu)]}=b_{y}\left[\frac{1}{x}-\frac{x}{4 h^{2}+x^{2}}-\frac{8 h^{2} x}{\left(4 h^{2}+x^{2}\right)^{2}}+\frac{4 h^{2} x^{3}-48 h^{4} x}{\left(4 h^{2}+x^{2}\right)^{3}}\right]+ \\
b_{x}\left[-\frac{24 h^{3} x^{2}-32 h^{5}}{\left(4 h^{2}+x^{2}\right)^{3}}\right]
\end{gathered}
$$

Comparing the above to eqs. (10-11), the components $K_{\imath \jmath}(\theta)$ are

$$
\begin{aligned}
& K_{x x}(\theta)=-\frac{\theta}{4 h^{2}+\theta^{2}}+\frac{8 h^{2} \theta}{\left(4 h^{2}+\theta^{2}\right)^{2}}+\frac{4 h^{2} \theta^{3}-48 h^{4} \theta}{\left(4 h^{2}+\theta^{2}\right)^{3}} \\
& K_{x y}(\theta)=-\frac{24 h^{3} \theta^{2}-32 h^{5}}{\left(4 h^{2}+\theta^{2}\right)^{3}} \\
& K_{y y}(\theta)=-\frac{\theta}{4 h^{2}+\theta^{2}}-\frac{8 h^{2} \theta}{\left(4 h^{2}+\theta^{2}\right)^{2}}+\frac{4 h^{2} \theta^{3}-48 h^{4} \theta}{\left(4 h^{2}+\theta^{2}\right)^{3}} \\
& K_{y x}(\theta)=\frac{24 h^{3} \theta^{2}-32 h^{5}}{\left(4 h^{2}+\theta^{2}\right)^{3}}
\end{aligned}
$$

The solution for the coordinates $\tilde{x}, \tilde{y}$ of the Erdogan 1969 and 1973 papers is then correctly given, using the transformation $K_{\tilde{x} \tilde{y}}(\theta)=-K_{x y}(\theta)$, $K_{\tilde{y} \tilde{x}}(\theta)=-K_{y x}(\theta)$, and $K_{\tilde{x} \tilde{x}}(\theta)=K_{x x}(\theta), K_{\tilde{y} \tilde{y}}(\theta)=K_{y y}(\theta)$, as

$$
K_{\tilde{x} \tilde{y}}(\theta)=-K_{\tilde{y} \tilde{x}}(\theta)=\frac{24 h^{3} \theta^{2}-32 h^{5}}{\left(4 h^{2}+\theta^{2}\right)^{3}}
$$

with on-diagonal $K_{\tilde{\imath} \jmath}(\theta)$ as above, whereas previously Erdogan (1969) stated $K_{\tilde{x} \tilde{y}}(\theta)=K_{\tilde{y} \tilde{x}}(\theta)=\left(24 h^{3} \theta^{2}-32 h^{5}\right) /\left(4 h^{2}+\theta^{2}\right)^{3}$. Erdogan (1971), using the same coordinates, is in agreement with these $K_{\tilde{\imath} \tilde{\jmath}}(\theta)$ provided the sign is 
changed for each of his kernel terms. That is appropriate for $K_{\tilde{x} \tilde{x}}$ and $K_{\tilde{y} \tilde{y}}$ because he reversed the sign convention for these terms in the 1971 paper, but not for $K_{\tilde{x} \tilde{y}}$ and $K_{\tilde{y} \tilde{x}}$. If we speculate that such was a misprint and that he intended to reverse the sign convention for the off-diagonal terms too, then results of his 1971 paper would be fully verified.

\section{References}

Ashbaugh, N., 1975. Stress solution for a crack at an arbitrary angle to an interface. Int. J. Fracture 11, 205-219.

Chen, S.H., Keer, L.M., Achenbach, J.D., 1980. Steady motion of a crack parallel to a bond-plane. Int. J. Eng. Sci. 18, 225-238.

Dundurs, J., Sendecky, G.P., 1965. Behavior of an edge dislocation near a bimetallic interface. J. Appl. Phys. 36, 3353-3354.

Dyskin, A.V., Germanovich, L.N., Ustinov, K.B., 2000. Asymptotic analysis of crack interaction with free boundary. Int. J. Solid. Struct. 37, 857-886.

Erdogan, F., 1969. Solutions of systems of singular integral equations. Soc. Ind. Appl. Math. J. Appl. Math. 17, 1041-1059.

Erdogan, F., 1971. Bonded dissimilar materials containing cracks parallel to the interface. Engineering Fracture Mechanics 3, 231-240.

Erdogan, F., Gupta, G.D., Cook, T.S., 1973. Numerical solutions of singular integral equations, in: Sih, G.C. (Ed.), Mechanics of Fracture: Methods of Analysis and Solution of Crack Problems. Noordhoff Int. Pub., Leyden, Netherlands. chapter 7, pp. 368-425.

Head, A.K., 1953. Edge dislocations in inhomogeneous media. Proc. Phys. Soc. B 66, 793-801.

Higashida, Y., Kamada, K., 1982. Stress fields around a crack lying parallel to a free surface. Int. J. Fracture 19, 39-52.

Murakami, Y. (Ed.), 1987. Stress Intensity Factors Handbook. volume 1. Pergamon Press, New York. 
Paynter, R.J.H., Hills, D.A., Sackfield, A., 2006. The penny crack beneath the surface of a half-space: The penny crack beneath the surface of a halfspace: with application to the blister test. Int. J. Fracture 142, 173-182.

Tada, H., Paris, P.C., Irwin, G.R. (Eds.), 2000. The Stress Analysis of Cracks Handbook. ASME, New York. 3rd edition.

Thouless, M.D., Evans, A.G., Ashby, M.F., Hutchinson, J.W., 1987. The edge cracking and spalling of brittle plates. Acta. Metall. 35, 1333-1341.

Yang, F., Li, J.C.M., 1997. Dislocation model of a subsurface crack. J. Appl. Phys. 82, 4816-4822. 